\title{
High-speed Detection Method of Whistler Dispersion for Determination of the Global Electron Density Distribution in the Ionosphere
}

\author{
Masashi Yoshida, Iwane Kimura, and Koji Kamejima \\ Graduate School of Information Science and Technology, Osaka Institute of Technology (OIT) \\ Kitayama, Hirakata, Osaka 573-0171, Japan \\ E-mail:m1m04a29@info.oit.ac.jp
}

\begin{abstract}
This research describes a high-speed method, which detects automatically whistler dispersion, to be used for determination of a global electron density distribution in the ionosphere and plasmasphere. It is required in order to know the detailed state of plasma.

It is necessary to observe the electron density distribution in the ionosphere in a short time scale, a day or shorter. A Japanese scientific satellite, "Akebono (EXOS-D)" has been used for various observations in the ionosphere and plasmasphere. The electron density distribution in these areas can be estimated from the propagation delay time of the whistler phenomenon, which is generated by electric discharge of the lightning on the ground and is observed by the satellite, the procedure to determine the distribution is to find a theoretically deduced model in which calculated propagation delay time fits the observed delay time.

The purpose of this study is to reduce the analysis time of the delay (dispersion) of whistlers observed by Akebono, using a pattern recognition technique, so called "Hough Transform"
\end{abstract}

\section{Introduction}

It is known that the radio wave propagation through the space is affected by the ionospheric plasma. The ionosphere is a plasma-state in which atoms and molecules are ionized. A radio wave propagates in vacuum at the velocity of light $\mathrm{c}$. But, in plasma it is refracted and its group velocity becomes smaller than $\mathrm{c}$ under the influence of plasma. The effect of ionospheric plasma depends on the wave frequency. VLF radio waves are strongly affected, and this effect cannot be disregarded even in higher frequencies, such as those used for the GPS system. Actually, the delay time of GPS signals increases a little due to the effect of plasmas and this additional delay time causes an error in the navigation system.
The electron density in the ionosphere is not always constant in time, and depends upon the local time, latitude, longitude, season and solar activity etc. In order to know the effect of the ionosphere to the GPS navigation system, the determination of a global electron density distribution is required in the time scale of a day or several hours at least.

One method to estimate a global ionospheric electron density profile by using Akebono VLF wave data was proposed [1]. That is to use the propagation delay time of the Omega signals observed by the Japanese scientific satellite "Akebono".

The Omega signals are those transmitted at around $10 \mathrm{kHz}$ for navigation support, so that most energy of the signals propagates in space between the lower boundary of the ionosphere and ground. A part of the energy passes through the ionosphere and can reach the satellite altitudes. An Omega signal observed by a satellite suffers from an additional delay due to ionospheric plasma.

A global electron density distribution can be determined from the delay time of the Omega signals observed along an Akebono satellite trajectory. Namely, a theoretical model of the plasmaspheric electron density distribution, with variable parameters are modified in such a way by ray tracing to give rise to the delay time obtained by the satellite observation.

Since the Omega system was terminated in 1997, it was intended to use the whistler phenomenon in place of the Omega signals observed by Akebono. Whistlers have frequency-time spectra, from which the propagation delay time can be determined [2], so that the whistler spectra can play a role to replace the Omega signal for the determination of the global electron density in the ionosphere and plasmasphere.

The propagation delay time of a whistler is determined as "dispersion" from its spectra. A method to obtain the whistler dispersion automatically, using a computer, from the VLF spectra observed by Akebono has been 
examined. The purpose of this study is to reduce the analysis time of whistler dispersion from the VLF spectral data.

\section{Spectral characteristic of whistler : Dispersion}

The whistler phenomenon is a radio noise, generated by an electric discharge of lightning on the ground, and is transmitted to space. Since there is a frequency dependency of the propagation velocity under the effect of electrons, in the ionosphere and plasmasphere, it has the feature that the time reaching an observing point differs in frequency. The whistler spectra observed by the satellite show a feature (frequency versus time) as shown in Figure-1.

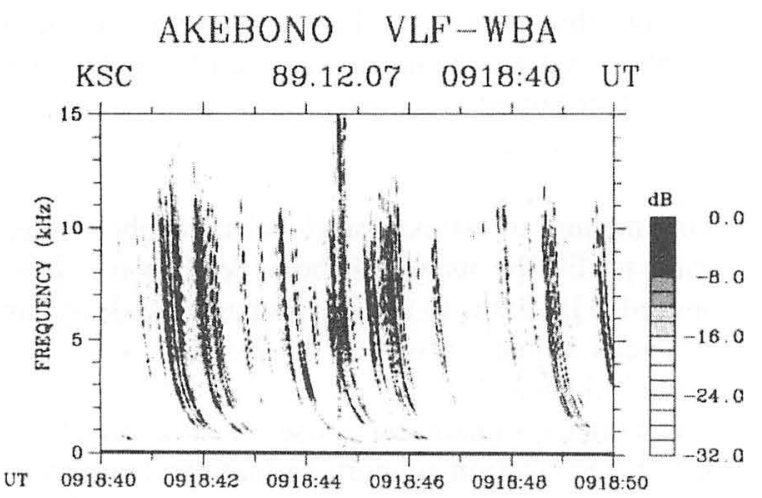

Fig-1 An example of the whistler spectra (Horizontal axis: universal time; hour:min:sec)

It is known that the propagation time $t[s]$ for a frequency $\mathrm{f}[\mathrm{Hz}]$ constituting a whistler is expressed by the following formula [3].

$$
t=\frac{D}{\sqrt{f}}
$$

$\mathrm{D}$ in this formula is called the dispersion of whistlers which is a quantity representing the integration of the electron density along the ray path. If $1 / \sqrt{f}$ is written as a new parameter $F$, the above formula can be transformed into a linear form.

$$
t=D F
$$

By using observed whistler spectra, the important quantity $\mathrm{D}$ (dispersion) that is a measure of the delay time, can be determined as the gradient of the above linear formula.

\section{[Program for signal processing]}

In the program of data processing to obtain the dispersion $\mathrm{D}$, the whistler spectra that were already A-D transformed in frequency [f] and time [t] domain are transformed into the straight-line on the F-t plane. During this process, the signal intensity is binary coded above and below a certain threshold level. Then the detection of the whistlers is made by finding a straight-line on this plane and the dispersion D is obtained from the gradient of the straight-line.

\section{High-speed detection method of Whistler dispersion}

A process to detect the whistlers from their spectra is automated by using pattern recognition technology of Hough Transform [4].

\subsection{The principle of Hough Transform}

This method uses an algorithm, i.e. Hough Transform that detects basic figures, such as a straight-line and an ellipse, from the binary image. The process of detection is divided into two; "voting" and "detection".

\section{1) Voting process}

For one pixel on the binary image of an F-t spectrum, we draw a straight-line passing through the pixel. The angle of this line with the time axis is $\theta$ and the distance from the origin to the line is denoted as $\rho$. Then this straight-line is transformed to a point on $\theta-\rho$ plane. For the initial pixel, many lines with different value of $\theta$ can be drawn. Each straight-line corresponds to a point on the $\theta-\rho$ plane, so that all possible straight-line are shown as a continuous curved-line on the $\theta-\rho$ plane. This operation is preformed for all pixels in the binary image.

On a computer, the values of parameters, $\theta$ and $\rho$ are quantized according to the detection accuracy. Number of voting in each quantized box on the $\theta-\rho$ plane is counted by accumulator. This accumulator is called voting matrix.

\section{2) Detection process}

In the accumulator after the voting process, an actual straight-line in the original binary image corresponds to a quantized box on the $\theta-\rho$ plane, where many counts are concentrated.

\section{[Computation tasks of Hough Transform]}

In the computational complexity of straight-line detection using Hough Transform, the times necessary for the operation of voting process is proportional to the number of lines at each pixel, and it is dependent on the quantized accuracy of the number of elements $N_{F}$ in the binary image, and total number of pixels (voting 
matrix) in the $\theta-\rho$ plane, i.e. $N_{\theta} \times N_{\rho} \cdot N_{\theta}$ and $N_{\rho}$ are related to the accuracy of detection in $\theta$ and

$\rho$. The time necessary for the detection process becomes larger for an increase of accuracy of quantization of a parameter. Both the computational complexities is $O\left(n^{2}\right)$. Namely,

$$
\begin{array}{ll}
\text { Voting Process } & N_{F} \times N_{\theta} \\
\text { Detection Process } & N_{\theta} \times N_{\rho}
\end{array}
$$

\subsection{Structure of voting matrix}

The detection time used in Hough Transform depends on efficiency of vote, and the structure of voting matrix.

When a voting matrix is securable on a memory in 2-dimensional arrangement, the computational complexity of count-up of the accumulator is $O(1)$. Since many memory domains are needed when the accuracy of quantization of parameter is high, it cannot secure a necessary size of the physical memory, and greatly affects on the processing time.

In this program, the voting matrix of the hash structure by 2-dimensional arrangement and the multi-dimensional list structure are used together. Although a search of the count-up in elements of the accumulator is needed at voting, the memory domains and the time of a detection process can be shortened when the voting matrix of list structure is used. When there are few elements in binary image, it is more advantageous in the total detection time than the voting matrix of the hash by 2-dimensional arrangement.

\subsection{High-speed algorithm of Hough Transform}

This section describes an increase of the efficiency by voting. A conventional program adopts a standard method of Hough Transform. In addition to this program, three high-speed algorithms by the following three different approaches are mounted.

\section{1) Probabilistic Hough Transform (PHT)}

This method extracts, at random, the pixels used for voting among the elements of binary image by the pre-assigned rate, and reduces the total computational complexity of straight-line detection.

Although the detection time is shortened if the rate that reduces the number of pixel in the image is decreased, the detection accuracy falls since the number of specimens used for detection decreases. This algorithm is an effective method when there are too many elements in binary image.

\section{[Rough estimation of the amount of computation]}

The number of operation in the voting process in this algorithm depends on the number of elements $N_{f}$, which is reduced from the number of elements of the binary image $N_{F}$, at a rate $R$, which is pre-assigned. Although the computational complexity $O\left(n^{2}\right)$ does not change, the total calculation time is shortened, since a coefficient is reduced as follows.

$$
N_{f} \times N_{\theta}
$$

The number $N_{f}$ is found by the following formula.

$$
N_{f}=R \times N_{F} \quad(0<R \leq 1)
$$

\section{2) Combinatorial Hough Transform (CHT)}

In this method, two pixels are selected in the binary image, the parameter of the straight-line, which passes through these two points, is calculated and it is counted in the accumulator.

This is efficient, because only the straight-lines that actually exist are enumerated. Since the processing time is only slightly affected by the accuracy of quantization, it is advantageous, when the analysis accuracy is high and there are few elements in the binary image.

\section{[Rough estimation of the amount of computation]}

The operation times of this algorithm is the combination of two pixels in image.

$$
{ }_{N_{F}} C_{2}=\frac{N_{F}\left(N_{F}-1\right)}{2}
$$

\section{3) Randomized Hough Transform (RHT)}

This method is a combination of the previous two algorithms. Namely, two pixels from the element of the binary image are selected by specimen extraction, and the parameter of the straight-line, which passes through the two points, is calculated, and it is counted in the accumulator.

This algorithm records the number of votes of straight-line parameters on 1-dimensional list structure. In this method, all parameters are recorded on a single list, so that the search time of a count-up element is longer as compared with the voting matrix of the multi-dimensional list structure.

Although the accuracy of detection is lower than other two algorithms, it is one effective method to detect simply the rough position of a straight-line.

\section{[Rough estimation of the amount of computation]}

The number of operations in the voting process of this algorithm is represented by. 


$$
{ }_{N_{f}} C_{2}=\frac{N_{f}\left(N_{f}-1\right)}{2}
$$

The number of operations is the number of combination of two pixels from the number of elements $N_{f}$ of binary image, which is reduced by specimen extraction.

\subsection{Comparison of the algorithms}

The performance of the standard method adopted by the conventional program, is compared with the high-speed algorithms of the newly mounted Hough Transform. The detection time of the straight-lines depends on the number of elements extracted by binarizing, and the accuracy of quantization of the parameter $(\theta-\rho)$. The processing time of each algorithm for these conditions is shown in Fig-2. The rate of specimen extraction $R$ is set as 0.6 .

As for the accuracy of quantization of the parameters as shown in Fig-2 a), the algorithm that uses the method of combination of pixels is not much affected by analysis accuracy. The factor that the processing time of RHT is longer than CHT depends on the efficiency of the record method of a voting matrix.

From the point of the number of elements of binary image, Fig-2 b) shows that PHT is the best. The reduction of detection time for PHT results from the specimen extraction, thought the voting approach is the same for PHT and SHT. This figure also shows that CHT and RHT are greatly affected by an increase of the number of elements.

\section{Detection from the spectrum data}

The observation data of the VLF band obtained from Akebono satellite have already been FFT (Fast Fourier Transform) processed, and are stored as spectral data of a long time scale. In the program, this spectrum is cut out to short time scale specimens, the order of 10 seconds, for analysis.

The detection time of whistler from a 10 second spectrum depends on the number of elements of the pixel in this time range, say 10 seconds. The number of elements of the pixel extracted by binarizing depends on the threshold. This threshold is determined by the discriminational analysis method, which calculates the threshold by statistical calculation.

Fig-3 shows the number of binarized elements in the spectrum of a 10 second specimen. This is just one example of the observation data. From this example, it is found that a large number of elements are involved even in a 10 seconds spectra.

\subsection{Choice of the best algorithm}

From the result of comparison of processing time, it is clarified that there is an appropriate algorithm for an assigned accuracy of quantization of parameters or the conditions of the number of elements in image. The time required for whistler detection can be shortened by selecting the appropriate method according to the

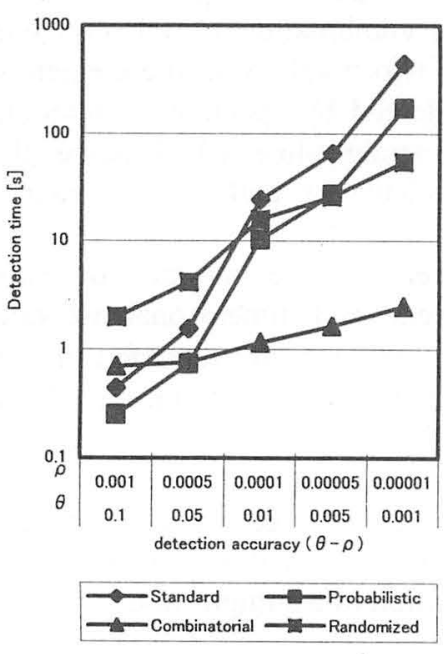

a) The detection time vs. detection accuracy

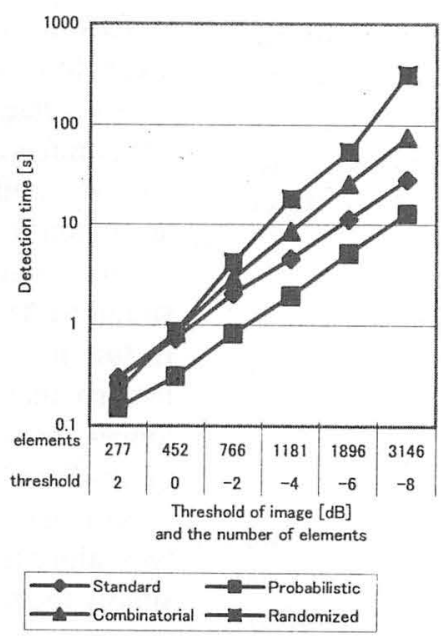

b) The detection time

vs. the number of elements

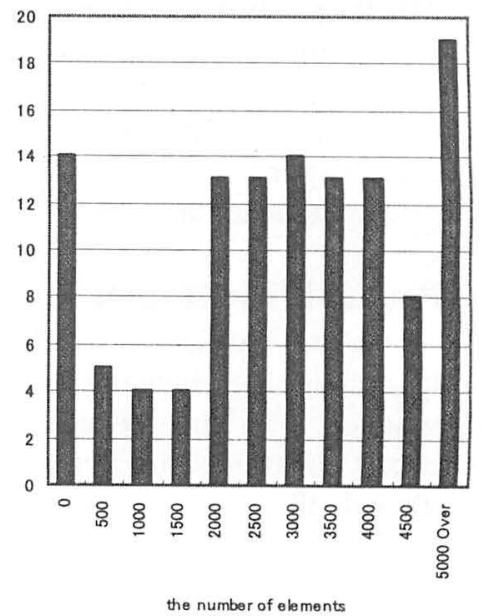

Fig-3 Distribution of the number of elements in 10 seconds specimens 
conditions of the image for a given time interval.

The method of the following standards and two kinds of high-speed methods are compared for the selection of algorithms.

- Standard Hough Transform (SHT)

- Probabilistic Hough Transform (PHT)

- Combinatorial Hough Transform (CHT)

It is required that the program, which detects whistlers and calculates their dispersion, should have a high accuracy. Therefore, it is necessary to select the best algorithm, in which, as much number of pixels should be used as possible for listing the line.

Although SHT and CHT, which do not extract specimen from the original data, are both suitable with reference to the accuracy of detection. The standard algorithm is better, since the specimen involves full number of elements.

CHT is used when an accuracy exceeding a default is needed. When there are many elements in the binary image, the algorithm that used CHT is not appropriate. But, the data processing time will be shorter than that method of enumerating all possible straight-lines passing through a pixel even if there are many elements when the accuracy of quantization of a parameter is high.

PHT is used when there are many elements. In these algorithms, specimen extraction must be performed for suppressing an increase of the processing time due to the number of elements of binary image.

RHT is the algorithm suitable for estimating the position of a rough straight-line. In the detection for which high accuracy is needed, it does not fit our objective. However the combined use of the algorithms may become useful after rough detection, for specifying the parameters of a detailed straight-line.

\subsection{Choice of the appropriate structure of Matrix}

The structure of a voting matrix should also be selected according to the conditions, together with algorithms. The memory structure is selected by the following two types.

- 2-dimensional arrangement

(Hash)

- Multi-dimension list

When the accuracy of quantization of a parameter is high, the list is more suitable than the hash from the point of detection time as a while. When the required accuracy of analysis is high or the number of elements of binary image is small, the list type is chosen unconditionally, or else the hash type is better.

\subsection{Results of the detection of whistler dispersion}

Fig- 4 is the detection time for long-duration spectral data. Moreover, Fig-5 a) and b) are the number of times of the algorithm and a voting matrix selected by the automatic judging.

The conventional method is using only SHT and the list type matrix. The improved method switches three algorithms and is using the voting matrix of two types.

It is possible to reduce the detection time by selecting the algorithms and the voting matrix automatically, according to the conditions.

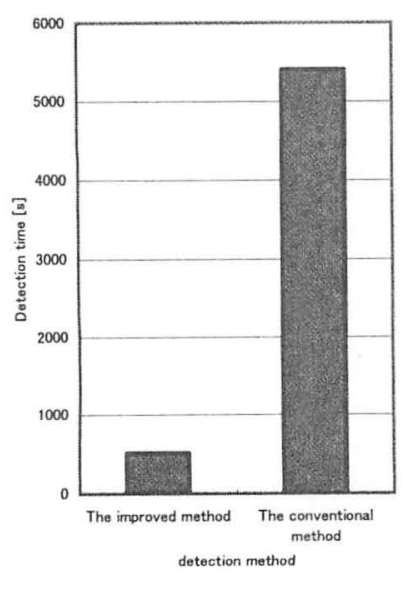

Fig-4 Comparison of the detection time of spectral data of a long time scale

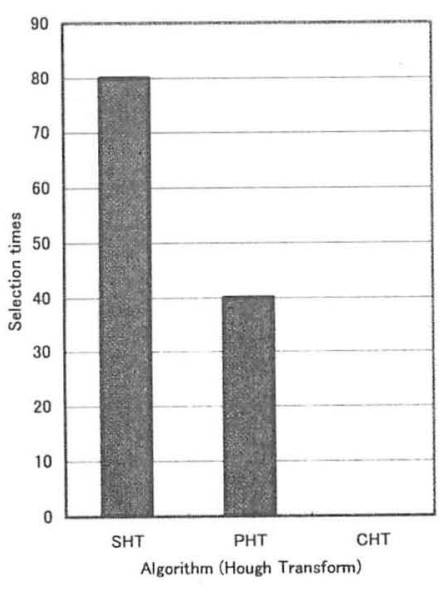

a) Selection times of algorithm

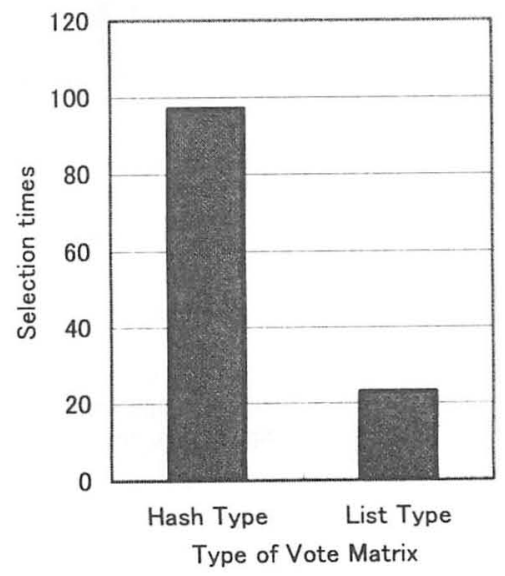

b) Selection times of type of vote matrix

Fig-5 The number of selection times vs. algorithms and type of voting matrix 


\section{Selection of right straight-line from Voting Matrix}

For assuring a high accuracy to detect the whistler dispersion the correctness of vote, and the method of picking up the optimal straight-line from a voting matrix, must be taken into account.

The parameters of straight-lines around the point, where many elements are concentrating in image, usually gain a large number of voting. Especially, in the area around a whistler with strong signal intensity, high voting is concentrated on the voting matrix.

In order to remove such false detection around the strong whistlers, a special filtering was devised. Namely at first, line-candidates are sorted out for the number of votes exceeding a certain threshold. We can exclude straight-lines crossing that of the highest number of votes within a pre-assigned frequency range.

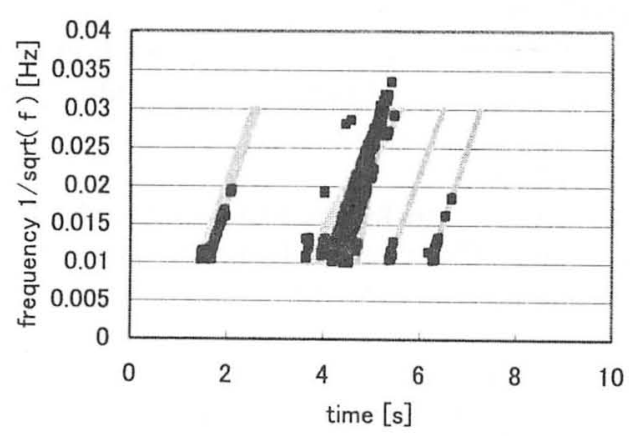

a) Result of default detection

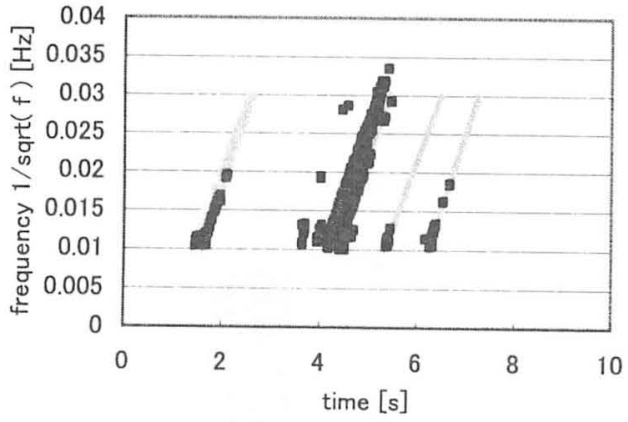

b) The effect of filtering

Fig-6 The detection result of the straight-line (whistler)

using Hough Transform

[Frequency vs. observed time]

Fig-6 is a comparison of the detection result of whistler by filtering in the spectrum for a 10 seconds specimen. False detection can be excluded with this filtering.

\section{Conclusion}

The program developed by this study tremendously reduced the analysis time for automatic detection of the whistler dispersion $\mathrm{D}$, and the necessary time of analysis becomes shorter than the elapsed time of the whistler phenomena on the observed VLF spectral. This study is expected to contribute to the ionospheric research.

\section{References}

[1] I.Kimura, K.Tsunehara, A.Hikuma, Y.Z.Su, Y.Kasahara, and H.Oya, Global electron density distribution in the plasmasphere deduced from Akebono wave data and the IRI model, J.Atmosope. Solar Terr. Phys 59(13), 1569,1997

[2] T.Shimizu: A study on automatic detection method for the dispersion of whistlers, master thesis OIT. ,2002.2

[3] K.Maeda and I.Kimura : Modern electromagnetism wave motion theory, Ohm Pub Co. , 1984.4

[4] R.Mochary and M.Shteiman : Computer Vision Course Hough Transform, 1998.11 